\title{
Microscopic Acid Dissociation of the 1:1 Copolymer of Methacrylic Acid and 2-Vinylpyridine
}

\author{
Seizo Masuda, ${ }^{\dagger}$ Keiji Minagawa, Masanori Tsuda, and Masami Tanaka* \\ Department of Chemical Science and Technology, Faculty of Engineering, \\ The University of Tokushima, Tokushima 770-8506, Japan \\ * Faculty of Pharmaceutical Science, Tokushima Bunri University, \\ Tokushima 770-8514, Japan
}

(Received March 30, 1998)

\begin{abstract}
Copolymerizations of methacrylic acid (MA) and methyl methacrylate (MMA) with 2-vinylpyridine (2VP) were carried out at $60^{\circ} \mathrm{C}$ with $2,2^{\prime}$-azobis(isobutyronitrile) (AIBN) as the initiator. Monomer reactivity ratios were obtained as $r_{1}=0.24$ and $r_{2}=0.46$ for the MA $\left(\mathrm{M}_{1}\right)-2 \mathrm{VP}\left(\mathrm{M}_{2}\right)$ system, and $r_{1}=0.46$ and $r_{2}=1.13$ for the MMA $\left(\mathrm{M}_{1}\right)-2 \mathrm{VP}\left(\mathrm{M}_{2}\right)$ system, respectively. The $1: 1$ copolymers of MA-2VP and MMA-2VP were prepared on the basis of monomer reactivity ratios obtained. Macroscopic and microscopic acid dissociation constants of the $1: 1$ copolymer of MA and 2VP were determined by pH-titration method. The relative concentrations of respective microscopic forms were calculated using the relation between micro-constants and hydrogen ion concentration. The fraction of the protonated form of the $1: 1$ copolymer of MA and $2 \mathrm{VP}$, which exists exclusively in the lower $\mathrm{pH}$ range, decreases with increasing $\mathrm{pH}$ value and fully vanishes at $\mathrm{pH}=6$, while the deprotonated form begins to appear at $\mathrm{pH}=5$. The zwitterionic and neutral molecule forms coexist in the $\mathrm{pH} 2$ to 10 region, and the latter form is predominant regardless of $\mathrm{pH}$ value. Macro- and micro-constants were also measured for the systems of the monomer mixture and the homopolymer mixture. Similar $\mathrm{pH}$-distribution curves are obtained for three systems (the $1: 1$ copolymer, the $1: 1$ homopolymer mixture, and the $1: 1$ monomer mixture)

KEY WORDS Methacrylic Acid / 2-Vinylpyridine / 1:1 Copolymer / Microscopic Acid Dissociation / pH-Distribution of Microscopic Forms /
\end{abstract}

In general, amino acids exist in protonated and deprotonated forms in acidic and basic regions, respectively, while there are coexistence of zwitterionic and neutral forms in neutral $\mathrm{pH}$-region. Such a behavior that microscopic forms are dependent on $\mathrm{pH}$ is one of the characteristics of amphoteric compounds.

Macroscopic and microscopic acid dissociation constants of a number of amphoteric compounds (for example, amino acids, ${ }^{1,2}$ oligopeptides, ${ }^{3,4}$ pyridine derivatives, ${ }^{5,6}$ and drugs ${ }^{7,8}$ ) were determined in relation to the dependence of pharmacological and biological activities on the degree of ionization.

Microscopic acid dissociation of the amphoteric polymer having both amino and carboxyl groups is interesting in connection with bioactive polymers. However, there is no report on microscopic acid dissociation of synthetic polymers in spite of many works on macroscopic acid dissociation of polyelectrolytes. ${ }^{9-12}$

From a structural point of view, poly(2-aminoacrylic acid $)^{13}$ is the most suitable synthetic polymer sample for determining microscopic acid dissociation. However, it is insoluble in water. A polymer having pyridine ring and carboxyl group as pendant is also an amphoteric compound, in which a proton of carboxyl group can migrate to nitrogen atom of the pyridine ring (interconversion of neutral molecule form to zwitterionic form). In the previous paper, ${ }^{14}$ the authors reported macroscopic and microscopic acid dissociation of the alternating copolymer of acrylic acid (AA) and 4vinylpyridine (4VP). When two monomers (AA and $4 \mathrm{VP}$ ) were mixed, half or above of molecules changed to the ionic form, and the copolymerization took place spontaneously to give the alternating copolymer with

\footnotetext{
† To whom all correspondence should be addressed.
}

relatively low molecular weight. On the other hand, no spontaneous copolymerization of methacrylic acid (MA) and 2-vinylpyridine (2VP) took place. The random copolymer of MA and 2VP was obtained using radical initiator.

The present paper is concerned with preparation of the 1:1 water soluble copolymer of $\mathrm{MA}$ and $2 \mathrm{VP}$ and determination of its macroscopic and microscopic acid dissociation constants. As a reference copolymer, the $1: 1$ copolymer of methyl methacrylate (MMA) and 2VP was also prepared. In addition, the distributions of microscopic forms of the 1:1 random copolymer of MA and 2VP are compared with those of the $1: 1$ alternating copolymer of AA and 4VP. In what follows, the $1: 1$ copolymers of MA with 2VP and MMA with 2VP are abbreviated as PACP and PMCP, respectively.

\section{EXPERIMENTAL}

\section{Materials}

Monomers and other reagents were commercially available and purified by standard methods prior to use.

\section{Copolymerization Procedure}

Copolymerizations of MA with 2VP in ethanol and MMA with $2 \mathrm{VP}$ in benzene were carried out at $60^{\circ} \mathrm{C}$ in sealed tubes with 2,2'-azobis(isobutyronitrile) (AIBN) as the initiator. Monomers, initiator, and solvent were placed into the tube and degassed by successive freezepump thaw cycles. After a fixed time, the contents were brought together with a large amount of ether so that copolymer precipitated. The copolymer was filtered off, dried under reduced pressure, and weighed. Copolymer composition was determined by ${ }^{1} \mathrm{H}$ NMR 


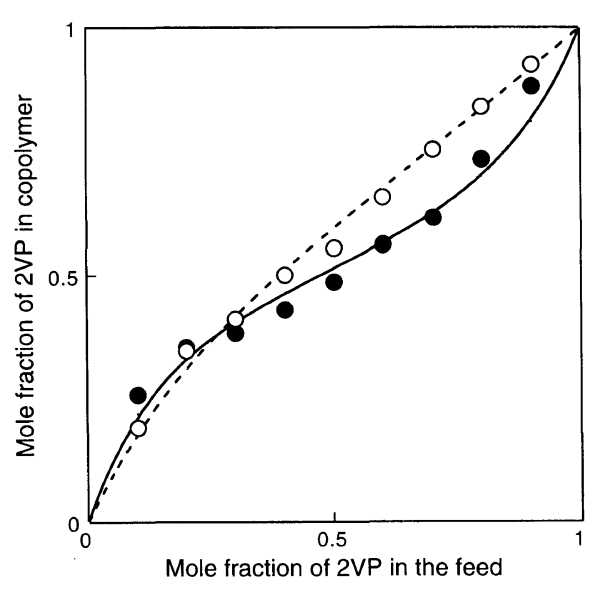

Figure 1. Monomer-copolymer composition curves for the MA-2VP (O) and MMA-2VP (O) systems.

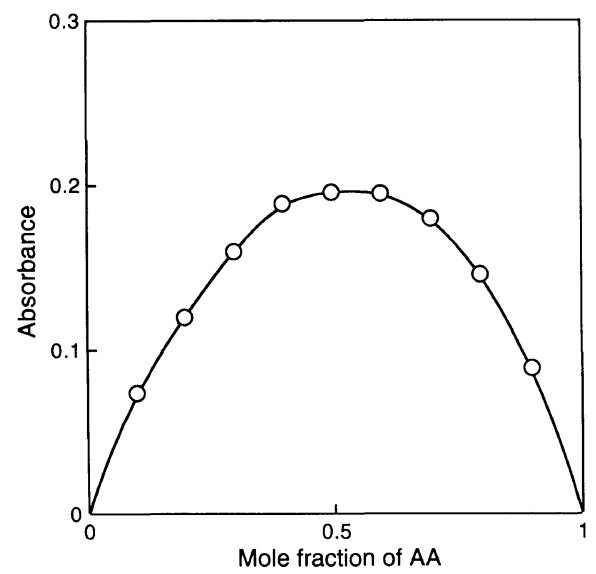

Figure 2. Continuous variation method in UV spectra for the MA and $2 \mathrm{VP}$ system. $[\mathrm{MA}]+[2 \mathrm{VP}]=1.0 \times 10^{-3} \mathrm{moll}^{-1}$; wave length, $300 \mathrm{~nm}$.

spectroscopy. Monomer reactivity ratios were calculated by the Kelen-Tüdös method. ${ }^{15}$

In order to determine acid dissociation constants, the $1: 1$ copolymers were prepared on the basis of monomer reactivity ratios obtained. The $1: 1$ copolymers obtained were purified by dialyzation and lyophilization.

\section{pH-Titration}

The $1: 1$ copolymer (PACP) was dissolved in distilled water, the concentration being $2.5 \times 10^{-3} \mathrm{moll}^{-1}$. The calculated amount of nitric acid equivalent to the content of vinylpyridine units in the copolymer was added to the solution, followed by potassium nitrate to keep the ion strength $(\mu=0.1)$. The solution was then titrated with $0.02 \mathrm{~N}$ potassium hydroxide. Monomer mixture, homopolymer mixture, and PMCP were also titrated.

\section{Measurements}

${ }^{1} \mathrm{H}$ NMR spectra were obtained with a JEOL GX-400 spectrometer, using deuterated water and tetramethylsilane (TMS) as the solvent and standard, respectively. UV spectra were measured on a Shimadzu UV-3000 spectrometer, ethanol being used as the solvent. $\mathrm{pH}$ Measurements were made at $25^{\circ} \mathrm{C}$ with a TOA HS$60 \mathrm{~S} \mathrm{pH}$-meter equipped with an automatic titrator.

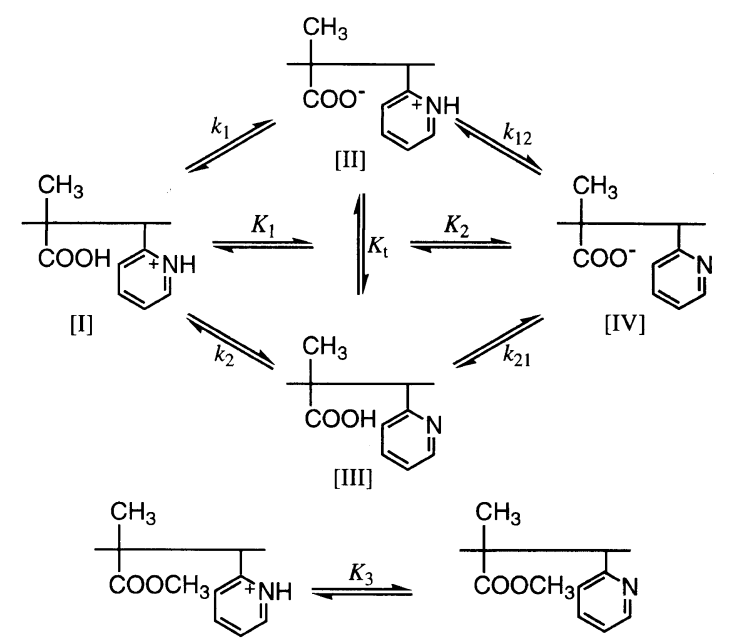

Figure 3. A schematic representation of macroscopic and microscopic ionization equilibria of PACP

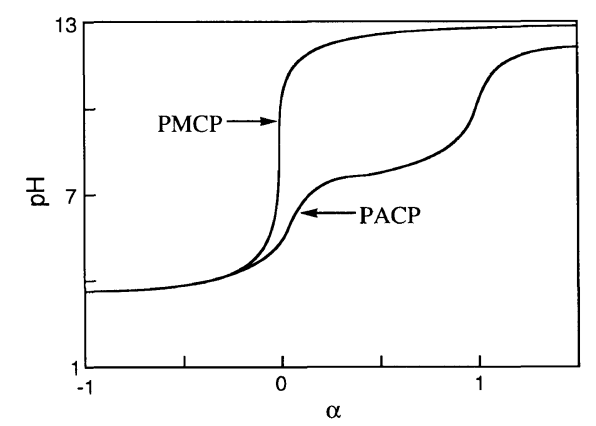

Figure 4. $\mathrm{pH}$-Titration curves of PACP and PMCP

\section{RESULTS AND DISCUSSION}

Copolymerizations of MA with 2VP in ethanol and MMA with $2 \mathrm{VP}$ in benzene were carried out at $60^{\circ} \mathrm{C}$ with AIBN as the initiator. Figure 1 shows monomercopolymer composition relationship. Monomer reactivity ratios were evaluated as $r_{1}=0.24$ and $r_{2}=0.46$ for the MA $\left(\mathrm{M}_{1}\right)-2 \mathrm{VP}\left(\mathrm{M}_{2}\right)$ system, and $r_{1}=0.46$ and $r_{2}=$ 1.13 for the MMA $\left(\mathrm{M}_{1}\right)-2 \mathrm{VP}\left(\mathrm{M}_{2}\right)$ system, respectively. On the other hand, monomer reactivity ratios were calculated as $r_{1}=0.36$ and $r_{2}=0.93$, for the MA $\left(\mathrm{M}_{1}\right)$-2VP $\left(\mathrm{M}_{2}\right)$ system and $r_{1}=0.40$ and $r_{2}=1.28$ for the MMA $\left(\mathrm{M}_{1}\right)-2 \mathrm{VP}\left(\mathrm{M}_{2}\right)$ system, respectively, using $Q$ and $e$ values described in literature $\left(Q_{\mathrm{MA}}=0.98\right.$, $e_{\mathrm{MA}}=0.62, Q_{2 \mathrm{VP}}=1.41, e_{2 \mathrm{VP}}=-0.42, Q_{\mathrm{MMA}}=0.78$, and $\left.e_{\mathrm{MMA}}=0.40\right) .{ }^{16}$ Comparison of found and calculated monomer reactivity ratios suggests that the MA-2VP copolymer obtained in this work exhibits higher alternating tendency than predicted by the literature $Q$ and $e$ values, while the MMA-2VP copolymer exhibits nearly the same reactivity ratios as predicted. This means there is appreciable interaction between MA and 2VP.

Figure 2 shows continuous variation method in UV $(300 \mathrm{~nm})$ for the MA-2VP system. There appears maximum absorption at $[\mathrm{MA}] /[2 \mathrm{VP}]=1$, which is indicative the formation of $1: 1$ complex. This complex seems to be ionic pair of 2-vinylpyridinium cation and gegen anion from methacrylic acid.

In order to determine microscopic constants, $1: 1$ copolymers PACP and PMCP were prepared on the basis 
Table I. Macroscopic and microscopic acid dissociation constants

\begin{tabular}{|c|c|c|c|c|c|c|c|}
\hline Sample & $\mathrm{p} K_{1}$ & $\mathrm{p} K_{2}$ & $\mathrm{p} k_{1}$ & $\mathrm{p} k_{2}$ & $\mathrm{p} k_{12}$ & $\mathrm{p} k_{21}$ & $K_{\mathrm{t}}$ \\
\hline PACPa & 3.71 & 7.64 & 4.51 & 3.79 & 6.84 & 7.56 & 0.19 \\
\hline Homopolymer mixture $^{b}$ & 3.70 & 7.23 & 4.21 & 3.86 & 6.72 & 7.07 & 0.45 \\
\hline Monomer mixture ${ }^{\mathrm{c}}$ & 4.25 & 5.53 & 4.88 & 4.36 & 4.90 & 5.42 & 0.30 \\
\hline
\end{tabular}

${ }^{a} 1: 1$ copolymer of methacrylic acid and 2-vinylpyridine. ${ }^{b}$ Equimolar mixture of poly(methacrylic acid) and poly(2-vinylpyridine). ${ }^{c}$ Equimolar mixture of methacrylic acid and 2-vinylpyridine.

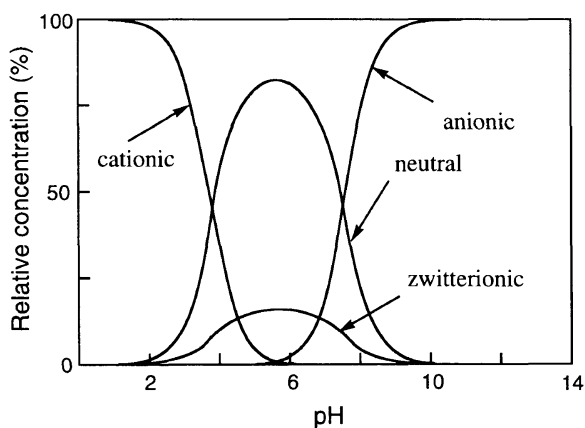

Figure 5. $\mathrm{pH}$-Distribution curves for the microscopic forms of PACP.

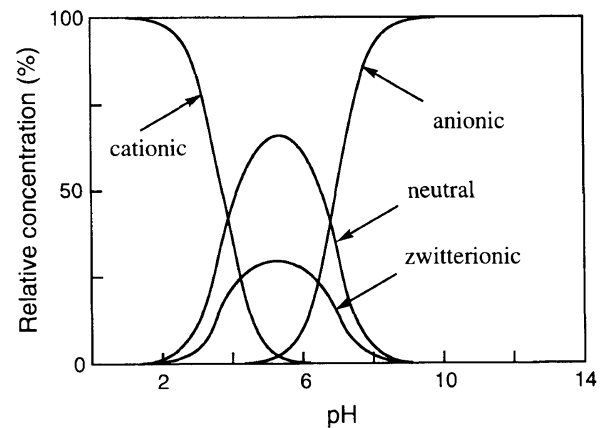

Figure 6. $\mathrm{pH}$-Distribution curves for the microscopic forms of the equimolar mixture of poly(methacrylic acid) and poly(2-vinylpyridine).

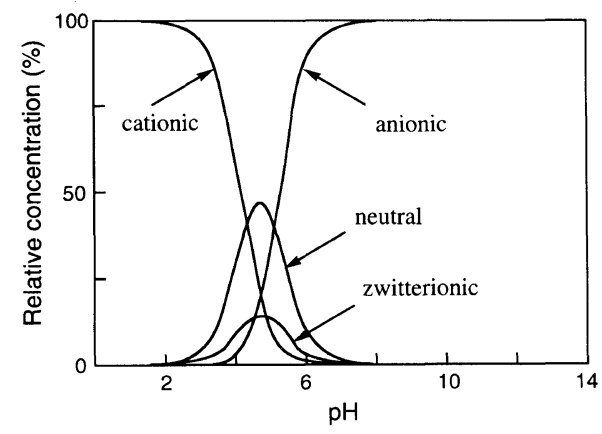

Figure 7. $\mathrm{pH}$-Distribution curves for the microscopic forms of the equimolar mixture of MA and 2VP.

of monomer reactivity ratios obtained. After purified by dialyzation and lyophilization, 1:1 composition was confirmed by ${ }^{1} \mathrm{H}$ NMR technique. Figure 3 gives a schematic representation of microscopic ionization equilibria of PACP, together with more familiar macroscopic stepwise acid distribution sequence. Various methods are known for determination of microscopic acid dissociation constants. ${ }^{17-21}$ Titration method was used in this study. Typical titration curves are shown in Figure 4. Symbol $\alpha$ (corresponding to degree of neutralization) is defined as follows. At $\alpha=-1$, nitrogen atoms on pyridine ring of PACP are perfectly quarternized by nitric acid added. A total amount of nitric acid added is neutralized at $\alpha=0$, and the carboxyl groups of PACP is perfectly dissociated at $\alpha=1$.

Macroscopic constants $\mathrm{p} K_{1}, \mathrm{p} K_{2}$, and $\mathrm{p} K_{3}$ were determined from the values of $\mathrm{pH}$ at $\alpha=-0.5,0.5$, and -0.5 , respectively. From the results of Figure 4, $K_{1}=1.95 \times 10^{-4}$ and $K_{2}=1.05 \times 10^{-8}$ for copolymer PACP and $K_{3}=1.62 \times 10^{-4}$ for PMCP were obtained. The relations between macroscopic and microscopic dissociation constants are as follows.

$$
\begin{aligned}
& k_{1}=\frac{\left[\mathrm{H}^{+}\right][\mathrm{II}]}{[\mathrm{II}]} \\
& k_{2}=\frac{\left[\mathrm{H}^{+}\right][\mathrm{III}]}{[\mathrm{I}]} \\
& k_{12}=\frac{\left[\mathrm{H}^{+}\right][\mathrm{IV}]}{[\mathrm{II}]} \\
& k_{21}=\frac{\left[\mathrm{H}^{+}\right][\mathrm{IV}]}{[\mathrm{III}]} \\
& K_{1}=\frac{\left[\mathrm{H}^{+}\right]\{[\mathrm{II}]+[\mathrm{III}]\}}{[\mathrm{II}]}=k_{1}+k_{2} \\
& \frac{[1}{K_{2}}=\frac{[\mathrm{II}]+[\mathrm{III}]}{\left[\mathrm{H}^{+}\right][\mathrm{IV}]}=\frac{1}{k_{12}}+\frac{1}{k_{21}} \\
& K_{1} \times K_{2}=\frac{[\mathrm{H}]^{2}[\mathrm{IV}]}{[\mathrm{II}]}=k_{1} \times k_{12}=k_{2} \times k_{21} \\
& K_{\mathrm{t}}=\frac{[\mathrm{II}]}{[\mathrm{III}]}=\frac{k_{1}}{k_{2}}=\frac{k_{21}}{k_{12}}
\end{aligned}
$$

By assuming $k_{2}=K_{3}$, microscopic constants $\left(k_{1}, k_{12}\right.$, and $k_{21}$ ) are calculated according to the above equations. This assumption is generally accepted because the dissociation equilibria of PMCP are essentially attributed to deprotonation of pyridinium ion. ${ }^{1}$ Table I summarizes acid dissociation constants and tautomeric constants $\left(K_{\mathrm{t}}\right)$. Inequalities of $\mathrm{p} k_{1}>\mathrm{p} k_{2}$, and $\mathrm{p} k_{21}>\mathrm{p} k_{12}$ apply to all systems. This shows that the neutral molecule form is superior to the zwitterionic form.

The distribution of the microscopic forms as a function of $\mathrm{pH}$ is of interest, in connection with $\mathrm{pH}$-dependence of biologically and physiologically active amino acids and pyridine derivatives. Figures $5-7$ show the fractional concentrations of the respective forms. The relative concentrations were calculated using the relationship equations, which are shown above, of microscopic constants and hydrogen ion concentration. The fraction of the cationic (protonated) form of PACP, which exists exclusively in the lower $\mathrm{pH}$ range, decreases with increasing $\mathrm{pH}$ value and fully vanishes at $\mathrm{pH}=6$, while 
the anionic (deprotonated) form begins to appear at $\mathrm{pH}=5$. The zwitterionic and neutral molecule forms coexist in the $\mathrm{pH} 2$ to 10 region, and the latter form is predominant regardless of $\mathrm{pH}$ value. $\mathrm{pH}$-Distribution curves for the monomer mixture and homopolymer mixture are similar to that for PACP. However, $\mathrm{pH}$ region, where zwitterionic and neutral molecule forms can coexist, becomes wider according to the order of monomer mixture $<$ homopolymer mixture $<1: 1$ copolymer.

There is no difference between the MA-2VP random copolymer and the AA-4VP alternating copolymer, considering from the standpoint of relative concentration of microscopic forms; i.e., the neutral form is superior to the zwitterionic form in both copolymers. However, they differ remarkably from each other in the shape of distribution curves. pH-Dependence of distributions of the neutral molecule and zwitterionic forms for PACP is relatively narrow and sharp curves. For the AA-4VP alternating copolymer, on the contrary, the neutral molecule and zwitterionic forms coexist over a wide range of $\mathrm{pH}(\mathrm{pH}=1-13)$ and the distribution gives curves with wide plateau.

It is generally known in amino acids such as glycine ${ }^{1}$ and lysine ${ }^{2}$ that zwitterionic form is more stable than neutral form in neutral region. We have also reported ${ }^{14}$ that the zwitterionic form is superior to the neutral form in the equimolar mixture of $\mathrm{AA}$ and $4 \mathrm{VP}$ monomers, but their alternating copolymer has opposite superiority. AA and 4VP were spontaneously copolymerized without any catalysts. The ionic pair or ionic complex of acrylic acid anion and 4-vinylpyridinium cation played an important role in this copolymerization.

On the other hand, the neutral form is predominant in the system of MA and 2VP, and no spontaneous copolymerization takes place because of low concentration of the ionic pair or ionic complex of acrylic acid anion and 4-vinylpyridinium cation. The reason for the preponderance of the neutral molecule type is probably that $\mathrm{MA}$ is a weaker acid than $\mathrm{AA}$ and $2 \mathrm{VP}$ is a weaker base than 4VP. Nevertheless, MA-2VP copolymer obtained in this work exhibits higher alternating tendency than predicted by the literature $Q$ and $e$ values. ${ }^{16}$

\section{REFERENCES}

1. J. T. Edsall and J. Wyman, "Biophysical Chemistry," Vol. 1, Academic Press, New York, N.Y., 1958, p 477.

2. D. L. Rabenstein and T. L. Sayer, Anal. Chem., 44, 114 (1976).

3. R. I. Shrager, J. C. Cohen, S. R. Heller, D. H. Sacks, and A. N. Schechter, Biochem., 11, 541 (1972).

4. D. L. Rabenstein, M. S. Greenberg, and C. A. Evans, Biochem., 16, 977 (1997).

5. S. F. Mason, J. Chem. Soc., 674 (1958).

6. K. Nakamoto and A. E. Martell, J. Am. Chem. Soc., 81, 5857 (1959).

7. H. Sakurai and T. Ishimitsu, Talanta, 27, 293 (1980).

8. W. H. Streng, J. Pharm. Sci., 67, 666 (1978).

9. S. Sugai and K. Nitta, "Kobunshi Denkaishitu," Kyoritsu Shuppan, Tokyo, 1978, p 41.

10. H. P. Gregor, L. B. Lutteinger, and E. M. Loebl, J. Phys. Chem., 59, 34 (1955).

11. M. Nagasawa, T. Murase, and K. Kondo, J. Phys. Chem., 69, 4005 (1965).

12. M. Nagasawa and A. Holtzer, J. Am. Chem. Soc., 86, 531 (1964).

13. R. S. Asquith, K. L. Gardner, and K. W. Yeung, J. Polym. Sci., 16, 3275 (1978)

14. S. Masuda, M. Tsuda, T. Tomida, and M. Tanaka, Chem. Lett., 601 (1994).

15. T. Kelen and F. Tüdös, J. Macromol. Sci., Chem., A9, 1 (1975).

16. J. Brandrup and E. H. Immergut, "Polymer Handbook," 3rd ed, John Wiley \& Sons, New York, N.Y., 1989, pp II-268-269.

17. T. Ishimitsu, H. Hinose, and Y. Fujiwara, Talanta, 26, 67 (1979).

18. G. E. Clement and T. P. Hartz, J. Chem. Educ., 48, 395 (1971).

19. H. L. Jung and L. Chang, J. Chem. Educ., 51, 106 (1974).

20. T. Ishimitsu, S. Hirose, and H. Sakurai, Talanta, 24, 555 (1977).

21. D. P. Wrathall, R. M. Izatt, and J. J. Christensen, J. Am. Chem. Soc., 86, 4779 (1964) 